\title{
Old hepatitis B virus never dies: It just hides itself within the host genome
}

\author{
Jeong-Hoon Lee \\ Department of Internal Medicine and Liver Research Institute, Seoul National University College of Medicine, Seoul, Korea
}

Keywords: Carcinoma, Hepatocellular; Hepatitis B surface antigens; Loss

See Article on Page 207

The World Health Organization (WHO) estimated that in 2015, 257 million people were living with chronic hepatitis B (CHB). Chronic infection with hepatitis B virus (HBV) is a major global health problem that resulted in an estimated 887,000 deaths in 2015, mostly due to hepatocellular carcinoma (HCC) and the complications of liver cirrhosis. The increased use of potent antivirals has gradually reduced HBV-related mortality in Korea. However, the decreased mortality has been paradoxically accompanied by the increased burden of HBV-related HCC, because of the increased life expectancy and number of patients with the risk of developing HCC.'

A three-tiered approach has been used to prevent or reduce the risk and mortality of HBV-related HCC: primary, secondary, and tertiary preventions. First, primary prevention entails protection from being infected by HBV, including being infected by vertical transmission. This can be mostly accomplished by active and passive immunization via recombinant $\mathrm{HBV}$ vaccine and hepatitis $B$ immunoglobulin, respectively. In 1992, the WHO recommended including universal HBV vaccination in all national vaccination programs. Every infant should receive the first dose of HBV vaccine as soon as possible after birth (preferably within 24 hours). Recent data have shown that the addition of tenofovir treatment to active and passive immunization might minimize the risk of mother-to-child transmission from an HBV surface antigen (HBsAg)-positive mother with a high HBV viral load at the third trimester. ${ }^{2}$ All international practice guidelines have emphasized the importance of primary prevention via vaccine and immunoglobulin with/without oral antiviral agents. ${ }^{3-6}$ Primary prevention should be the most cost-effective and ideal approach to the prevention of HBV-related HCC, especially in HBV-endemic regions. Secondary prevention entails reducing the risk of HCC occurrence in patients who already have chronic HBV infection and is based on the use of potent oral nucleos(t)ide analogues (NAs) such as entecavir and tenofovir. There are two pathways involved in the development of HCC in patients with CHB: 1) the direct oncogenic effect of HBV (direct hepatocarcinogenesis) and 2) an indirect effect through inflammation, regeneration, fibrosis, and cirrhosis (indirect hepatocarcinogenesis). An effective lifelong antiviral treatment might reduce useless and harmful hepatic inflammation

\begin{abstract}
Abbreviations:
$\mathrm{CHB}$, chronic hepatitis $\mathrm{B}$; $\mathrm{HBeAg}$, hepatitis $\mathrm{B}$ virus envelope antigen; $\mathrm{HBs} A$ g, hepatitis $B$ virus surface antigen; HBV, hepatitis B virus; $H C C$, hepatocellular carcinoma; NAs, nucleos(t)ide analogues; WHO, World Health Organization
\end{abstract}

\section{Corresponding author : Jeong-Hoon Lee}

Department of Internal Medicine and Liver Research Institute, Seoul National University College of Medicine, 101 Daehak-ro, Jongno-gu, Seoul 03080, Korea

Tel: +82-2-2072-2228, Fax: +82-2-743-6701

E-mail: pindra@empal.com or JHLeeMD@snu.ac.kr

https://orcid.org/0000-0002-0315-2080 
and thereby suppress the indirect pathway. Several cohort studies conducted in Asian countries have consistently reported that timely antiviral treatment reduced the risk of HCC by $45-63 \%$. $^{7-10}$ Although tenofovir-resistant mutations have been observed in several heavily treated patients, ${ }^{11}$ the incidence of tenofovir-resistant mutations has been negligible in patients who were not previously exposed to other antivirals. Thus, antiviral resistant mutation might not be problematic during lifelong antiviral treatment, but medical cost might. Tertiary prevention aims to reduce the risk of HCC recurrence and to prolong both the recurrence-free survival and overall survival of patients. Adjuvant therapy consisting of repeated transfer of autologous cytokine-induced killer cells ${ }^{12}$ as well as oral antiviral treatment has been found to play a role in the tertiary prevention of HCC and increased patient survival.

In this issue of Clinical and Molecular Hepatology, Jang et al. ${ }^{13}$ reported that the integration of HBV DNA into host genome was observed in the majority of seven patients who developed HCC after HBsAg seroclearance and were included in the present study. The affected genes were mostly associated with carcinogenesis. Viral integration breakpoints were detected in both malignant and adjacent nonmalignant tissues. Interestingly, the HBV insertional sites were more enriched within the genic region of the tumor sample rather than in intergenic area, whereas intergenic HBV integration was more frequent in the non-tumor tissues. This study confirmed again the possible HCC development even in patients who achieved HBsAg seroclearance. However, the finding that five of the seven (71.4\%) patients had underlying cirrhosis confounds the identification of which pathway among the indirect and direct pathways in these patients was the major route leading to hepatocarcinogenesis. Cirrhosis could be a more important cause of HCC than integrated HBV in those five cirrhotic patients.

The following two important questions could be derived from this study. First, is there a feasible method for preventing the integration of HBV DNA into host genome of patients already infected with HBV? And second, what is the optimal surveillance strategy for patients after HBsAg seroclearance?

The currently available anti-HBV treatments, including oral HBV DNA polymerase inhibitors and pegylated interferon-alfa, generally fail to achieve the elimination of integrated HBV DNA, which is considered to be a complete sterilizing cure of HBV infection. Thus, the presence of integrated HBV DNA is thought to indicate that a complete sterilizing cure may be unrealistic. Therefore, a discussion is warranted on the possibility of developing an approved therapy that could prevent the integration of HBV DNA into genomic DNA before it occurs. The timing of initiating antivi- ral treatment might be a modifiable factor. Studies by Mason et al. ${ }^{14}$ showed that HBV DNA integration into the host genome and clonal expansion of hepatocytes begin during the immune-tolerant phase. A recent study evaluating the effect of RNA interference by ARC-520 on HBsAg in chimpanzees chronically infected with HBV showed that the integration of HBV DNA into the host genome occurs during the HBV envelope antigen (HBeAg)-positive phase..$^{15}$ Based on these lines of evidence, evaluating whether early antiviral treatment at a specific timepoint before HBV DNA integration during the HBeAg-positive stage could reduce or prevent HBV DNA integration and subsequent clonal expansion of hepatocytes would be interesting. Since NAs inhibit HBV DNA polymerase, which also synthesizes double-stranded linear DNA, which integrates within the host genome, it may be possible that early treatment with an NA can reduce the risk of the integration of HBV DNA into the host genome. ${ }^{16}$ However, since few data supporting this hypothesis on early treatment are available, further study is warranted. Altogether, it dosen't seem that we currently possess a reliable weapon for preventing or eliminating HBV integration.

Next, the surveillance strategy in patients experiencing past HBV infections should be an important issue. It must be clear that based on the current guidelines, patients who have already developed cirrhosis at the time of HBsAg seroclearance should undergo regular surveillance by imaging studies (ultrasonography, computed tomography, or magnetic resonance imaging) and serum alpha-fetoprotein levels at 6 -month intervals. All international guidelines recommend regular surveillance for patients with cirrhosis due to any cause. ${ }^{3-6}$ In contrast, patients without obvious cirrhosis or advanced fibrosis who are identified by regular followups to have achieved HBsAg seroclearance may lie in the gray zone. However, they clearly have a higher risk of HCC development than normal individuals not exposed to HBV, but a lower risk than patients with $\mathrm{HBsAg-positivity} \mathrm{and} \mathrm{suppressed} \mathrm{serum}$ HBV DNA. ${ }^{17,18}$ Additionally, should a patient without a previous history of HBsAg positivity who is incidentally found at a health check-up to be positive for anti-HBV core lgG antibody and negative for both HBsAg and HBV DNA, which indicate resolved HBV infection need regular surveillance? No sufficient data to answer the question are currently available and precise stratification to select optimal target of regular surveillance needs to be defined considering cost-effectiveness. Baseline factors such as the presence of cirrhosis, duration of HBV infection, mode of HBsAg seroclearance (natural vs. antiviral-induced), coexisting metabolic risk factors (e.g., obesity, hypertension, dyslipidemia, and diabetes), 
and genotype of HBV might be associated with the risk of HCC development in patients with resolved HBV infection. ${ }^{17,19,20}$ The establishment of an HCC prediction model for patients with resolved HBV infection combining those risk factors might be warranted to guide personalized HCC surveillance program.

In summary, HBsAg seroclearance does not guarantee that HCC will not develop. The persistence of HBV integrated into the host genome despite the disappearance of serum HBsAg, along with the fibrotic burden due to previous injury to the hepatocytes, might be responsible for a residual risk of HCC. Since we do not have effective options for preventing integration of HBV DNA after infection, the prevention of HBV infections by universal vaccination cannot be emphasized too strongly. HCC surveillance of individuals achieving HBsAg seroclearance should be individualized, and further studies to establish a model of risk prediction are warranted.

\section{Conflicts of Interest}

The author has no conflicts to disclose.

\section{REFERENCES}

1. Choi J, Han S, Kim N, Lim YS. Increasing burden of liver cancer despite extensive use of antiviral agents in a hepatitis B virus-endemic population. Hepatology 2017;66:1454-1463.

2. Pan CQ, Duan Z, Dai E, Zhang S, Han G, Wang Y, et al. Tenofovir to prevent hepatitis $B$ transmission in mothers with high viral load. N Engl J Med 2016;374:2324-2334.

3. Terrault NA, Lok ASF, McMahon BJ, Chang KM, Hwang JP, Jonas $M M$, et al. Update on prevention, diagnosis, and treatment of chronic hepatitis B: AASLD 2018 hepatitis B guidance. Hepatology 2018;67:1560-1599.

4. European Association for the Study of the Liver. EASL 2017 clinical practice guidelines on the management of hepatitis B virus infection. J Hepatol 2017;67:370-398.

5. Sarin SK, Kumar M, Lau GK, Abbas Z, Chan HL, Chen CJ, et al. Asian-Pacific clinical practice guidelines on the management of hepatitis B: a 2015 update. Hepatol Int 2016;10:1-98.

6. Korean Association for the Study of the Liver (KASL). KASL clinical practice guidelines for management of chronic hepatitis B. Clin Mol Hepatol 2019;25:93-159.

7. Wong GL, Chan HL, Mak CW, Lee SK, Ip ZM, Lam AT, et al. Entecavir treatment reduces hepatic events and deaths in chronic hepatitis
B patients with liver cirrhosis. Hepatology 2013;58:1537-1547.

8. Su TH, Kao JH. Response to four-year entecavir therapy reduces hepatocellular carcinoma, cirrhotic events and mortality in chronic hepatitis B patients. Liver Int 2017;37:310-311.

9. Wu CY, Lin JT, Ho HJ, Su CW, Lee TY, Wang SY, et al. Association of nucleos(t)ide analogue therapy with reduced risk of hepatocellular carcinoma in patients with chronic hepatitis B: a nationwide cohort study. Gastroenterology 2014;147:143-151.e5.

10. Hosaka T, Suzuki F, Kobayashi M, Seko Y, Kawamura Y, Sezaki H, et al. Long-term entecavir treatment reduces hepatocellular carcinoma incidence in patients with hepatitis B virus infection. Hepatology 2013;58:98-107.

11. Park ES, Lee AR, Kim DH, Lee JH, Yoo JJ, Ahn SH, et al. Identification of a quadruple mutation that confers tenofovir resistance in chronic hepatitis B patients. J Hepatol 2019;70:1093-1102.

12. Lee JH, Lee JH, Lim YS, Yeon JE, Song TJ, Yu SJ, et al. Adjuvant immunotherapy with autologous cytokine-induced killer cells for hepatocellular carcinoma. Gastroenterology 2015;148:1383-1391.e6.

13. Jang JW, Kim JS, Kim HS, Tak KY, Nam H, Sung PS, et al. Persistence of intrahepatic hepatitis $B$ virus DNA integration in patients developing hepatocellular carcinoma after hepatitis B surface antigen seroclearance. Clin Mol Hepatol 2021;27:217-218.

14. Mason WS, Gill US, Litwin S, Zhou Y, Peri S, Pop O, et al. HBV DNA integration and clonal hepatocyte expansion in chronic hepatitis B patients considered immune tolerant. Gastroenterology 2016;151:986-998.e4.

15. Wooddell $\mathrm{Cl}$, Yuen MF, Chan HL, Gish RG, Locarnini SA, Chavez D, et al. RNAi-based treatment of chronically infected patients and chimpanzees reveals that integrated hepatitis B virus DNA is a source of HBsAg. Sci Transl Med 2017;9:eaan0241.

16. Yang W, Summers J. Integration of hepadnavirus DNA in infected liver: evidence for a linear precursor. J Virol 1999;73:9710-9717.

17. Kim GA, Lee HC, Kim MJ, Ha Y, Park EJ, An J, et al. Incidence of hepatocellular carcinoma after HBsAg seroclearance in chronic hepatitis B patients: a need for surveillance. J Hepatol 2015;62:1092-1099.

18. Yip TC, Wong GL, Chan HL, Tse YK, Lam KL, Lui GC, et al. HBsAg seroclearance further reduces hepatocellular carcinoma risk after complete viral suppression with nucleos(t)ide analogues. J Hepatol 2019;70:361-370.

19. Lee YB, Moon H, Lee JH, Cho EJ, Yu SJ, Kim YJ, et al. Association of metabolic risk factors with risks of cancer and all-cause mortality in patients with chronic hepatitis B. Hepatology. 2020 Nov 2. doi: 10.1002/hep.31612.

20. Chen YC, Sheen IS, Chu CM, Liaw YF. Prognosis following spontaneous HBsAg seroclearance in chronic hepatitis B patients with or without concurrent infection. Gastroenterology 2002;123:1084-1089. 